\title{
Gibt es einen Zusammenhang zwischen Bewegungsaktivität und psychischem Befinden im Alltag?
}

\section{Eine Methodenillustration zum ambulanten Monitoring in der Gesundheitspsychologie}

\author{
Andreas Schwerdtfeger ${ }^{1}$, Ragna Eberhardt ${ }^{1}$ und Andrea Chmitorz ${ }^{2}$ \\ ${ }^{1}$ Psychologisches Institut, Johannes Gutenberg-Universität Mainz \\ ${ }^{2}$ Department Psychologie, Ludwig-Maximilians Universität München
}

\begin{abstract}
Zusammenfassung. Mit diesem Beitrag wollen wir illustrieren, wie mit Hilfe der Technik des ambulanten Monitoring gesundheitspsychologische Fragestellungen im Alltag untersucht werden können. Dazu wurde der Zusammenhang von körperlicher Aktivität und Wohlbefinden im Alltag analysiert. Mit Hilfe von Beschléunigungssensoren wurden Bewegungsdaten über einen Zeitraum von 12 Stunden an 124 Probanden zwischen 18 und 73 Jahren aufgezeichnet. Energetische Aktiviertheit/ positiver Affekt (EA/PA) und Anspannung/negativer Affekt (WA/NA) wurden etwa stündlich mittels Pocketcomputer erfasst. Die Daten wurden mit Mehrebenenmodellen ausgewertet. Bewegungsepisoden, die vor der Befindensabfrage auftraten, waren positiv mit EA/PA assoziiert. Zusammenhänge mit WA/NA konnten hingegen nicht beobachtet werden. Weiterhin waren BMI und EA/PA negativ miteinander assoziiert, wobei dieser Zusammenhang vor allem auf die Männer zurückzuführen war. Weiterhin zeigte sich, dass mit zunehmendem BMI der Zusammenhang zwischen Bewegung und EA/PA anstieg. Die Ergebnisse zeigen, dass die Untersuchung gesundheitspsychologischer Fragestellungen im Feld viel versprechend ist und die Befunde bisheriger Untersuchungen erweitern.

Schlüsselwörter: Ambulantes Monitoring, Bewegungsaktivität, Befinden, energetische Aktiviertheit, wahrgenommene Anspannung
\end{abstract}

Is there a correlation between everyday-life physical activity and psychological well-being? A methodological study using ambulatory monitoring

Abstract. This study aimed to examine if a correlation exists between physical activity and mood in daily life using ambulatory monitoring technology. 124 individuals ( 64 females, age range $18-73$ years) participated. Physical activity was recorded throughout one day (12 hours) via accelerometers and mood (energetic arousal/positive affect and tension arousal/ negative affect) was assessed about every hour using handheld (palm) computers. Mixed model analyses revealed that energetic arousal/positive affect was significantly positively associated with preceding physical activity episodes. Moreover, BMI was negatively associated with energetic arousal/positive affect and this effect was especially pronounced in males. Further analyses showed that with increasing BMI the relation between physical activity and energetic arousal/positive affect grew stronger. Contrary to energetic arousal/positive affect tension arousal/negative affect was unrelated to physical activity. The results suggest that daily physical activity episodes seem to modulate mood aspects and that ambulatory monitoring technology is a useful tool for examining these associations.

Key words: ambulatory assessment, ecological momentary assessment, emotional well-being, mood, physical activity

\section{Theoretischer Hintergrund}

Ambulantes Monitoring beschreibt die ambulante (d.h. im Feld stattfindende) Erfassung psychologischer, behavioraler und/oder physiologischer Variablen (Fahrenberg, Leonhart \& Foerster, 2002; Fahrenberg, Myrtek, Pawlik \& Perrez, 2007). Das Hauptanliegen dieses Forschungsansatzes besteht in der Generalisierbarkeit von Befunden auf den Alltag von Personen; somit verfolgt diese Methodik ein zentrales Anliegen psychologischer Forschung.

Die u. a. von Fahrenberg et al. (2002, 2007) propagierte Methodik des ambulanten Monitoring bedient sich beispielsweise der Datenerfassung mittels Pocketcomputer und physiologischer Datenrekorder und erlaubt somit die parallele und z. T. auch interaktive Erfassung verschiedener Datenebenen über längere Zeiträume. Im Gegensatz 
zu rückblickenden Selbstbeurteilungen, wie sie recht häufig z. B. in Tagebuchstudien anzutreffen sind, können die so gewonnenen subjektiven Daten zeitlich genau über den Tagesverlauf hinweg registriert werden und unterliegen somit keinen retrospektiven Verzerrungen (siehe z.B. Käppler, Brügner \& Fahrenberg, 2001). Auch Verhaltensdaten, wie z. B. körperliche Bewegung können mittels Aufzeichnungssystemen (z.B. Beschleunigungssensoren) objektiv erfasst werden (z.B. Foerster \& Fahrenberg, 2000), wobei subjektive Verzerrungen und mögliche Fehleinschätzungen seitens der Probanden vermieden werden. Ein weiterer Vorteil dieses Ansatzes besteht in der Möglichkeit, intraindividuelle Kovariationen zwischen behavioralen oder physiologischen Variablen einerseits und subjektiven Daten andererseits zu analysieren sowie interindividuelle Moderatoren eines solchen Zusammenhangs zu identifizieren.

Die vorliegende Studie hat zum Ziel, die ambulante Methodik anhand einer häufig untersuchten Fragestellung innerhalb der Gesundheitspsychologie zu illustrieren, nämlich der Frage nach dem Zusammenhang von körperlicher Bewegung und psychischem Befinden. Bevor wir auf die Methodik im Einzelnen eingehen, wollen wir den bisherigen Forschungsstand auf diesem Gebiet kurz skizzieren.

\section{Körperliche Aktivität und psychisches Befinden}

Es existieren mittlerweile zahlreiche Untersuchungen über den Zusammenhang von körperlicher Aktivität und psychologischen Befindensvariablen. Die meisten dieser Studien wurden dabei in mehr oder weniger streng kontrollierten Settings (z. B. Sportkurse, Trainingseinheiten) durchgeführt. Eine zusammenfassende Beurteilung der Befunde scheint schwierig, da viele Studien methodische Mängel aufweisen (z. B. keine adäquaten Kontrollgruppen), unterschiedliche Stichproben heranzogen (Patienten vs. Gesunde) oder verschieden intensive Arten körperlicher Aktivität berücksichtigten (Gruppenprogramme oder Einzelprogramme unterschiedlicher Frequenz, Gerätetraining unterschiedlicher Intensität etc.). Es zeichnet sich jedoch ab, dass körperliche Aktivität und Wohlbefinden positiv miteinander assoziiert sind und entsprechend Bewegungsaktivität einen protektiven Faktor für die psychische Gesundheit darstellen könnte. Gleichwohl muss festgehalten werden, dass die Kausalität dieses Effekts noch nicht gesichert ist (für Meta-Analysen siehe z.B. Arent, Landers \& Etnier, 2000; Craft \& Landers, 1998; Puetz, O’Connor \& Dishman, 2006; Reed \& Ones, 2006; Schlicht, 1993).

Die Rolle psychologischer Korrelate körperlicher Aktivität gewinnt auch vor dem Hintergrund physischer Gesundheit zunehmend an Relevanz. In neuerer Zeit wird mitunter die Ansicht vertreten, dass auch weniger intensive körperliche Aktivität oder gar Alltagsaktivität der Aufrechterhaltung und Wiederherstellung der physischen Gesundheit dienlich sein soll, da sie als weniger aversiv beurteilt wird und somit die Wahrscheinlichkeit einer kontinuierlichen Ausführung erhöht ist (Abu-Omar \& Rütten, 2006; Ekkekakis, Hall, van Lanuyt \& Petruzzello, 2000; National Institutes of Health Consensus Development Panel on Physical Activity and Cardiovascular Health, 1996; Pate et al., 1995). Entsprechend versuchen neuere Studien, die günstigen psychologischen Effekte von kurzzeitiger und moderater körperlicher Aktivität aufzuzeigen (z.B. Hall, Ekkekakis \& Petruzello, 2002).

Psychologische Effekte lassen sich vor allem für die Variablen positiver Affekt (PA) oder wahrgenommene energetische Aktiviertheit (EA) absichern, wohingegen die Befundlage für negative Stimmungen weniger eindeutig ist (z. B. Otto \& Stemmann, 1991; Thayer et al., 2003). PA beschreibt das Erleben positiver Affekte wie z. B. Aktiviertheit, Interesse oder Begeisterung (siehe Watson, Clark \& Telegen, 1988). In ähnlicher Weise indiziert EA einen Zustand, der bei hoher Ausprägung mit Gefühlen von Tatkraft und Vitalität und bei niedriger Ausprägung mit Müdigkeit einhergeht (Thayer, 1978). Zur Erfassung dieser konzeptuell verwandten Dimensionen (siehe hierzu Yik, Russell \& Feldman Barrett, 1999) sind die „Positive and Negative Affect Schedule" (PANAS; Watson et al., 1988) oder die ,Activation-Deactivation-AdjectiveChecklist" (AD-ACL; Thayer, 1989) herangezogen worden.

Beide positiv gefassten Befindensvariablen sind abzugrenzen von den orthogonal dazu konzipierten Dimensionen negativer Affekt (NA; Watson et al., 1988) und wahrgenommene Anspannung (WA; Thayer, 1978). WA beispielsweise bezeichnet Zustände, die von Anspannung bis hin zu Gelassenheit und Ruhe variieren können (Thayer, 1978) und NA indiziert Affekte wie nervös, gereizt oder bekümmert (zur Orthogonalität von EA/PA und WA/NA siehe z. B. Yik et al., 1999).

In verschiedenen Feldstudien konnten Thayer, Peters, Takahashi und Birkhead-Flight (1993) belegen, dass kurze Spaziergänge von 4 bis 10 Minuten Dauer das Maß der wahrgenommenen Energie positiv beeinflussen und in einigen Fällen sogar Spannung und Müdigkeit reduzierten. Auch Ekkekakis, Hall, van Landuyt und Petruzzello (2000) konnten positive Effekte von kurzen Spaziergängen von 10 bis 15 Minuten Dauer auf EA und PA aufzeigen. Sie fanden allerdings keinen Einfluss von Bewegung auf die Variablen NA oder WA. Zusammenfassend scheint moderate körperliche Aktivität also primär mit Veränderungen in den positiven Aktivierungsvariablen EA und PA einherzugehen. Dieser Befund ist weitgehend kompatibel zu Meta-Analysen, die zeigen, dass die positive Aktiviertheit auch positiv mit aerober sportlicher Aktivität assoziiert ist (z. B. Reed \& Ones, 2006).

\section{Überleitung zur vorliegenden Untersuchung}

Zusammenfassend lässt sich also feststellen, dass moderate Episoden körperlicher Aktivität von relativ kurzer Dauer (4-15 Minuten) mit Veränderungen in positiver 
Aktiviertheit einhergehen können. Wir wollen nun der Frage nachgehen, in wie weit dieser Zusammenhang auch im Alltag nachweisbar ist, d.h. sind auch Bewegungsepisoden, die üblicherweise im Alltag ausgeführt werden (wie z. B. Treppensteigen, kurze Episoden von Gehen, Laufen oder Radfahren), mit Befindensveränderungen assoziiert? Erste Hinweise auf einen möglichen Zusammenhang liefert eine Studie von Thayer et al. (2003). Die Autoren konnten beobachten, dass das Ausmaß von körperlicher Bewegung, die im Alltag gezeigt wird (erfasst über Schrittzähler; sog. Pedometer), mit EA positiv assoziiert war, wohingegen kein Zusammenhang mit WA/NA gefunden werden konnte. Allerdings wurde in dieser Studie die Befindlichkeit nur alle 4 Stunden mittels Fragebogen erhoben, so dass eine detailliertere und methodisch stringente Analyse dieses Zusammenhangs nicht möglich war.

Zur Untersuchung des Bewegungs-Befindens-Zusammenhangs bedienten wir uns der Methodik des ambulanten Monitoring. Durch Einsatz von mobilen Geräten zur Erfassung des Befindens (z.B. Personal Digital Assistants; PDAs) und der Bewegungsaktivität (Beschleunigungssensoren) war es uns darüber hinaus möglich, den Einfluss der Dauer von Bewegungsepisoden hinsichtlich des Zusammenhangs mit Befindensvariablen näher zu untersuchen. So wurden in der vorliegenden Studie verschiedene Zeitfenster der alltäglichen Bewegungsaktivität (1 Minute, 5 Minuten, 15 Minuten) erfasst und mit den Befindensvariablen EA/PA und WA/NA in Beziehung gesetzt.

\section{Methode}

\section{Teilnehmer}

Die Erhebung wurde an 64 weiblichen und 60 männlichen Versuchspersonen $(n=124)$ mit einem relativ breiten Altersbereich zwischen 18 und 73 Jahren $(M=31.67, S D=$ 12.56) durchgeführt. ${ }^{1}$ Die Teilnehmer wurden über Aushänge an den Gebäuden der Universität Mainz und über mündliche Kontaktaufnahme rekrutiert. Entsprechend setzte sich die Stichprobe aus $66 \%$ Studierenden $(n=82)$ verschiedener Fachrichtungen, $23 \%$ Erwerbstätigen ( $n=$ 28), $1 \%$ Schülern $(n=1), 4 \%$ Erwerbslosen $(n=5)$ und $6 \%$ Ruheständlern $(n=8)$ zusammen. Der Body-Mass-Index (BMI) variierte zwischen 17.57 und $35.93 \mathrm{~kg} / \mathrm{m}^{2}(M=23.23$, $S D=3.14)$, d.h. in der Stichprobe befanden sich sowohl untergewichtige, normalgewichtige, als auch adipöse Personen (gemäß der Definition der Weltgesundheitsorganisation; WHO).

\section{Apparatur}

Aktivitätserfassung. Die Bewegungsaktivität im Alltag wurde mit Beschleunigungssensoren (Actigraph ${ }^{\circledR}$, Mo-

${ }^{1}$ Die hier verwendete Stichprobengröße ist deutlich größer als die in verschiedenen Publikationen zu Mehrebenenanalysen empfohlenen $n=50$ oder $n=100$ (siehe zusammenfassend Nezlek et al., 2006) und sollte somit zu reliablen Parameterschätzungen führen. dell GT1M) am Fußgelenk der Probanden gemessen (vgl. Guinhouya, Hubert, Dupont \& Durocher, 2005). Das Gerät wurde mittels eines Klettbandes unmittelbar oberhalb des Fußknöchels (kontralateral zur dominanten Hand) befestigt. Diese Lokalisation wurde gewählt, um Bewegungsaktivitäten der unteren Extremitäten (z. B. Gehen, Treppensteigen, Radfahren) mit hinreichender Sensitivität zu erfassen. Allerdings muss eingeräumt werden, dass durch die Wahl dieses Ableitortes auch irrelevante, bzw. im Sinne der Fragestellung unerwünschte Ereignisse, wie z.B. nervöses Fußwackeln, erfasst werden.

Das Gerät zeichnet Beschleunigungen der vertikalen Achse mit $30 \mathrm{~Hz}$ auf, hat die Maße $38 \times 37 \times 18 \mathrm{~mm}$ und ein Gewicht von $27 \mathrm{~g}$ und ist somit unauffällig zu tragen. Die abgeleiteten Beschleunigungssignale durchlaufen einen Bandpassfilter ( .25 bis $2.5 \mathrm{~Hz})$, dessen Frequenzbereich primär sensitiv für körperliche Bewegung ist und höhere Frequenzen (z. B. Vibrationen, die durch Motoren ausgelöst werden können) unterdrückt. Die Sensitivität des Gerätes liegt im Bereich von 0.05 bis $2 \mathrm{G}(\mathrm{G}=$ Gravitation/ Beschleunigung). Bewegung wird quantifiziert in Einheiten (sog. „,counts"). Dabei entspricht eine Einheit einer Aktivität von 4 milli Gs/Sekunde. Für die Interpretation der hier gewonnenen Beschleunigungswerte sollte beachtet werden, dass typische Alltagsaktivitäten wie langsames Gehen (mit $3 \mathrm{~km} / \mathrm{h}$ ) zu etwa 5000 Einheiten/Minute führen, Treppensteigen zu etwa 8000 Einheiten/Minute und Fahrradfahren zu etwa 15000 Einheiten/Minute. Mit zunehmender Beschleunigung, die auf den Fuß einwirkt, werden also höhere Werte erzielt.

Die Reliabilität von Beschleunigungssensoren (und insbesondere des hier eingesetzten Typs) kann gemeinhin als gut bezeichnet werden (z. B. Matthews, Ainsworth, Tompson \& Basset, 2002; Trost, Pate, Freedson, Sallis \& Taylor, 2000). Ebenso bestätigen verschiedene Untersuchungen die Validität von Beschleunigungssensoren, indem mäßig $(r=.49)$ bis hohe Korrelationen $(r=.91)$ (je nach Ableitort, laborexperimentellem Setting und Gerätetyp) mit dem Energieverbrauch bzw. der Sauerstoffaufnahme gefunden wurden (für einen Überblick siehe Trost, McIver \& Pate, 2005).

Psychisches Befinden. Die Aufzeichnung des psychischen Befindens erfolgte durch PDAs der Marke PalmOne ${ }^{\circledR}$, Modell Zire 31 (Maße $120 \times 85 \times 25 \mathrm{~mm}$ ), die mit der Software DialogPad (Gerhard Mutz, Universität Köln) programmiert waren. Die Erhebung des Befindens erfolgte durch die Abfrage von 14 Items mit einer Likertskala von 1-5 (gar nicht - äußerst). In Übereinstimmung mit früheren Studien (z. B. Ekkekakis et al., 2000) wurden die Adjektive teilweise aus der AD-ACL nach Thayer (1989; vgl. die deutsche Version von Imhof, 1998) sowie aus der deutschen Form des PANAS von Krohne, Egloff, Kohlmann und Tausch (1996) entnommen. Weitere Adjektive, die kompatibel mit der Konzeption einer positiven bzw. negativen Aktivierung waren, wurden ebenfalls hinzugenommen. Die Adjektive wurden in permutierter Reihenfolge dargeboten und vor der Analyse zwei Skalen zugeordnet: EA/PA und WA/NA. 
Zur Erfassung von EA/PA dienten die Adjektive aktiv (AD-ACL, PANAS), dynamisch (AD-ACL), schlapp (-) (AD-ACL), müde (-) (AD-ACL), lebhaft (AD-ACL), wach (PANAS) und leistungsfähig (eigene Überlegung); zur Erfassung von WA/NA dienten die Items zufrieden $(-)$ (eigene Überlegung), entspannt (-) (AD-ACL), nervös (AD-ACL, PANAS), bedrückt (eigene Überlegung), erfreut (-) (PANAS), gestresst (eigene Überlegung) und gereizt (PANAS). Die möglichen Punktwerte beider Skalen für jede Messung liegen somit zwischen 7 und 35. Für die Skala EA/PA ergab sich über alle Abfragen hinweg ein Mittelwert von $23.17(S D=5.83)$ und für die Skala WA/NA ein Mittelwert von $15.05(S D=4.89)$. Insgesamt fanden über alle Versuchspersonen 1745 Befindlichkeitsabfragen mittels PDA statt, wovon $83 \%$ (1455 Abfragen) vollständig beantwortet wurden, das entspricht im Mittel etwa 12 Abfragen pro Proband.

Um die Reliabilität des Instruments zu ermitteln, wurde die jeweils erste reguläre Befindensabfrage jedes Probanden zur Berechnung des Cronbach $\alpha$ herangezogen. Für die Variable EA/PA konnte ein Wert von .90 ermittelt werden und für die Variable WA/NA ein Wert von .74. Zu betonen ist, dass beide Variablen nur moderat negativ miteinander assoziiert waren, $(r=-.32, p<.01)$, was im Sinne zweier weitgehend orthogonaler Dimensionen interpretiert werden kann (siehe Yik et al., 1999). ${ }^{2}$

Zusätzlich wurde die Änderungssensitivität berechnet, die für die Erfassung intraindividueller Verläufe unabdingbar ist. Hierbei variierte die intraindividuelle Standardabweichung der Variable EA/PA zwischen 0.84 und 8.02. Für die Variable WA/NA wurde eine Variation von 0.79 bis 7.17 ermittelt. Um kurzfristige Schwankungen (von Messzeitpunkt zu Messzeitpunkt) besser abbilden zu können, wurde das mittlere Quadrat sukzessiver Differenzen gebil$\operatorname{det}$ (MQSD; siehe Ebner-Priemer, Linehan \& Bohus, 2006). Hierbei werden die Differenzen aufeinander folgender Beantwortungen gebildet, quadriert und gemittelt. Für die Variable EA/PA ergab sich eine Variation zwischen 1 und 89.22 (dies entspricht einer Variation auf der Skala von durchschnittlich 1 Punkt bis zu 9.45 Punkten von Messzeitpunkt zu Messzeitpunkt). Das MQSD für die Variable WA/NA variierte zwischen 1 und 122.30 (dies entspricht einer Variation auf der Skala von durchschnittlich 1 Punkt bis zu 11.06 Punkten von Messzeitpunkt zu Messzeitpunkt).

Fragebogen. Am Ende des Tages wurde von den Versuchspersonen ein Fragebogen bearbeitet. Dieser beinhaltete Angaben zu demographischen Daten (Alter, Geschlecht, Größe, Gewicht, Beruf/Studienfach) und zur Beurteilung der Typizität des vergangenen Tages (Skala von $1-4 ; 1=$,absolut typisch“, 2 = „,eher typisch“, 3 = „,eher

${ }^{2}$ Die Validität der Skalen wurde überprüft, indem Korrelationen mit retrospektiven Einschätzungen (für den gesamten Tag) auf der PANAS (PA und NA) analysiert wurden. Die Variable EA/PA war signifikant mit PA korreliert $(r=.56, p<.01)$ und WA/NA korrelierte signifikant mit der retrospektiven Einschätzung von NA $(r=.69, p<.01)$. untypisch“, 4 = „ganz und gar untypisch“). Darüber hinaus wurde nach Einschränkungen im Tagesverlauf durch die Versuchsteilnahme gefragt (Skala von 1-3; $1=$, ja, sehr stark", 2 = ,teilweise“, 3 = ,nur geringfügig bzw. gar nicht") sowie nach erlebten Beeinträchtigungen durch den Beschleunigungssensor (Skala von 1-3; 1 = ,ja, sehr stark", 2 = „teilweise“, 3 = „nur geringfügig bzw. gar nicht"). Schließlich erfolgte eine retrospektive Einschätzung, in wie weit die Probanden glaubten, an diesem Tag körperlich aktiv gewesen zu sein, auf einer visuellen Analogskala (VAS) von 0 (,völlig inaktiv“) bis 100 (,,andauernd aktiv").

\section{Versuchsablauf}

Die Datenerhebung fand an einem von der Versuchsperson gewählten Tag in einem Zeitraum von 12 Stunden zwischen $10 \mathrm{Uhr}$ und $22 \mathrm{Uhr}$ statt. Der PDA wurde zwischen 9 Uhr und 9.30 Uhr aktiviert, so dass die automatische Abfrage bis spätestens $11 \mathrm{Uhr}$ begann und nachfolgend regelmäßig im Abstand von ungefähr einer Stunde $(60 \pm 15 \mathrm{~min})$ erfolgte. Ein akustisches Signal forderte die Teilnehmer zur Eingabe der Daten auf. Die Zufallskomponente von \pm 15 min wurde gewählt, um eine Antizipation des Signals mit evtl. assoziierter Verhaltensanpassung auszuschließen. Sollte es der Versuchsperson nicht möglich gewesen sein auf das Signal zu reagieren, war eine manuelle Aktivierung zu einem späteren Zeitpunkt möglich. Vor Beginn der Erhebung wurden die Probanden in die Handhabung des Gerätes eingewiesen und eine Probeabfrage initialisiert. Nach Aushändigung der Geräte und des Fragebogens konnten die Probanden ihren täglichen Beschäftigungen nachgehen und wurden lediglich durch die etwa stündliche Abfrage ihres aktuellen Befindens und das Ausfüllen des Fragebogens nach 22 Uhr an die Versuchsteilnahme erinnert.

\section{Datenauswertung und -analysen}

Um die zeitliche Vergleichbarkeit der ambulanten Daten zu gewährleisten erfolgte wöchentlich eine zeitliche sekundengenaue Synchronisation von Actigraph ${ }^{\circledR}$ und PDA mittels eines Computerprogramms (TimeAdjust ${ }^{\circledR}$, Stevens Creek Software). Regelmäßige Kontrollen der Uhrzeit zeigten, dass die Uhrzeitdifferenz zwischen Actigraph ${ }^{\circledR}$ und PDA nach einer Woche nie größer als 7 Sekunden war. Der Beschleunigungssensor speichert minutenweise die gemessene Aktivität und versieht diese mit einem Zeitstempel. Ebenso speichert der PDA die Eingaben sowie die Uhrzeit des Beginns der Dateneingabe. In der Datenanalyse wurden die Daten des PDA ausgelesen und die Aktivität, die vor der Befindensabfrage gemessen wurde, extrahiert. Wir analysierten drei Zeitfenster der Bewegungsaktivität, nämlich 1 Minute, 5 Minuten und $15 \mathrm{Mi}$ nuten vor der Befindensabfrage. Die Zeitfenster der Aktivitätsmessung endeten also unmittelbar vor Beginn der Befindensabfrage und erstreckten sich über die entsprechenden Zeitintervalle (die Bewegungsaktivität in den 5 
und 15 Minuten-Zeitfenstern wurde relativiert an einer Minute, um eine bessere Vergleichbarkeit der Ergebnisse zu ermöglichen). Zusätzlich wurde auch die an diesem Tag gemessene Gesamtaktivität zwischen 10 und 22 Uhr berechnet.

Wie Schwartz und Stone (1998) betonen, erfordert die statistische Analyse von wiederholt im Alltag der Versuchspersonen erhobenen Daten (sog. ecological momentary assessment) die Berücksichtigung verschiedener methodischer Besonderheiten, die in der Datenstruktur begründet sind. So werden Daten häufig zu unterschiedlichen Erhebungszeitpunkten mit Zufallskomponente erfasst (sog. nichtstationäre Zeitreihen), und unvollständige Datensätze (nicht beantwortete Abfragen) sind recht häufig anzutreffen. Daher wurde die Analyse mittels Mehrebenenmodellen (so genannter mixed models oder hierarchischer linearer Modelle) durchgeführt, mit denen variierende serielle Abhängigkeiten, ungleiche Erhebungszeitpunkte und personenspezifische Effekte wesentlich besser modelliert werden können als mit den klassischen statistischen Verfahren (Keller, 2003; Nezlek, Schröder-Abé \& Schütz, 2006; Raudenbush \& Bryk, 2002). Mehrebenenmodelle erlauben es, intraindividuelle Zusammenhänge (within-person, Level 1) abzubilden, Gruppenvergleiche (between-person, Level 2) vorzunehmen sowie Interaktionen zwischen within- und between-person Variablen zu berechnen. Die Grundannahme dieser Modelle besteht darin, dass die Level 1-Regressionskoeffizienten selbst wieder zufällige Variablen sind, die sich durch die Level 2-Prädiktoren vorhersagen lassen.

Die Logik der Auswertung beruhte auf der Prädiktion der EA/PA bzw. WA/NA durch die Bewegungsaktivität (1, 5 und 15 Minuten vor der Befindensabfrage) (Level 1), die demographischen Variablen Alter (kontinuierlich), Geschlecht $(0=$ männlich, 1 = weiblich) und BMI (kontinuierlich) (Level 2) sowie durch Interaktionen der Level 2-Variablen und Interaktionen zwischen den Ebenen (Level $1 \times$ Level 2). Insgesamt wurden 6 Modelle gerechnet (2 Kriterienvariablen und 3 Zeitfenster). Modelle mit Zufallseffekten wurden berechnet (z. B. Nezlek et al., 2006; Raudenbush \& Bryk, 2002), und für die Schätzung der fixen Parameter wurde die Restricted Maximum Likelihood Methode (REML) verwendet. Folgende Formel veranschaulicht die Auswertungsstrategie für die Variable EA/PA:

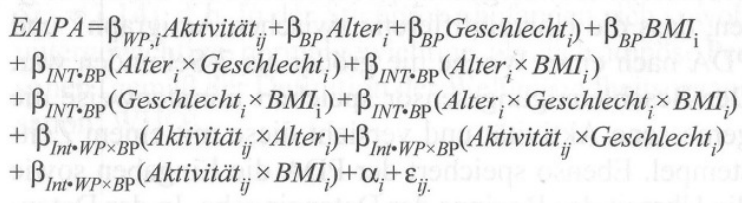

Hinweis: $\mathrm{WP}=$ within-person $($ Level 1$) / \mathrm{BP}=$ between-person (Level 2)/INT = Interaktionen

Auf Grund möglicher serieller Abhängigkeiten der Fehlervarianzen wurde eine autoregressive kontinuierliche Fehlerkorrektur für nichtstationäre Zeitreihen berechnet. Um die Steigungskoeffizienten besser interpretieren zu können, wurden die gemessenen Aktivitätswerte vor der Analyse durch 1000 dividiert. Des Weiteren wurden die kontinuierlichen Prädiktoren (Alter, BMI) zentriert, um die Interpretierbarkeit der Effekte zu gewährleisten. ${ }^{3}$ Die Datenauswertung erfolgte mit der Statistiksoftware R(Version 2.4.1; R Development Core Team, 2007), Paket „nlme“ (Pinheiro, Bates, DebRoy \& Sarkar, 2007). Das Signifikanzniveau wurde auf $p<.05$ (zweiseitige Testung) festgelegt.

\section{Ergebnisse}

\section{Fragebogendaten}

Zunächst wurde die Typizität des Tages analysiert und die subjektiv erlebte Einschränkung des Tagesablaufs durch die Versuchsteilnahme eruiert. Der Tag verlief für $66 \%$ der Probanden $(n=82)$ absolut typisch bzw. eher typisch, für $32 \%(n=40)$ verlief der Tag eher untypisch, und nur $2 \%(n=2)$ gaben an, einen ganz und gar untypischen Tagesverlauf gehabt zu haben. Weiterhin fühlten sich $94 \%$ der Probanden $(n=116)$ durch die Versuchsteilname gar nicht oder nur geringfügig in ihrem Tagesablauf eingeschränkt, während sich $6 \%(n=7)$ teilweise eingeschränkt fühlten und 1 Proband $(1 \%)$ sich sehr eingeschränkt fühlte. Schließlich fühlten sich durch die verwendeten Geräte $75 \%(n=93)$ der Versuchspersonen nur geringfügig bzw. gar nicht gestört, $24 \%(n=30)$ teilweise und 1 Proband $(1 \%)$ sehr stark.

Diese Befunde zeigen, dass eine Untersuchung im Alltag der Probanden mehrheitlich akzeptiert wird und den Tagesablauf kaum einschränkt. Allerdings ist es uns nur eingeschränkt gelungen, einen typischen Tagesablauf untersucht zu haben, da etwa 1/3 der Probanden den Tagesablauf als eher untypisch oder ganz und gar untypisch klassifizierten.

\section{Analyse der Bewegungsaktivität im Alltag}

Zunächst wurde die Korrelation zwischen der mittels Beschleunigungssensor gemessenen Gesamtaktivität über den Tag und der retrospektiven Selbsteinschätzung der körperlichen Aktivität (VAS 0-100) gerechnet. Beide Maße korrelierten signifikant positiv miteinander $(r=.41$, $p<.01)$. Dieses Ergebnis kann als Hinweis auf konvergente Validität beider Instrumente gedeutet werden. Gleichwohl muss festgehalten werden, dass die gemeinsame Varianz doch eher gering war, und entsprechend dürfen diese Maße nicht als äquivalent betrachtet werden. Weiterhin wurden Zusammenhänge zwischen den mit den Abfragen assoziierten Bewegungseinheiten in den drei Zeitfenstern (1 Minute, 5 Minuten und 15 Minuten vor Beginn der Befindensabfrage) und der über den Tag hinweg gemessenen Gesamtaktivität zwischen 10 und 22 Uhr

${ }^{3}$ Auf eine Zentrierung der Variable Bewegungsaktivität wurde verzichtet, da diese über einen natürlichen, interpretierbaren Nullpunkt verfügt. 
Tabelle 1. Mehrebenenmodelle zur Vorhersage von energetischer Aktiviertheit/positivem Affekt (EA/PA; links) und wahrgenommener Anspannung/negativem Affekt (WA/NA; rechts) im Alltag. Berichtet werden die Steigungskoeffizienten der Modelle $(b)$

\begin{tabular}{|c|c|c|c|c|c|c|c|}
\hline Kriterium/Prädiktor & $b^{1)}$ & $b^{5)}$ & $b^{15)}$ & Kriterium/Prädiktor & $b^{1)}$ & $b^{5)}$ & $b^{15)}$ \\
\hline$E A / P A$ & & & & $W A / N A$ & & & \\
\hline Level 1: Bewegungsaktivität & $.44 * *$ & $.45^{*}$ & $.58 * *$ & Level 1: Bewegungsaktivität & .03 & .10 & .21 \\
\hline Level 2: Alter & $.08 \dagger$ & $.08 \dagger$ & $.08 \dagger$ & Level 2: Alter & $-.07 *$ & $-.07 *$ & $-.06 \dagger$ \\
\hline Geschlecht $(0=\mathrm{m}, 1=\mathrm{w})$ & $-1.48 \dagger$ & $-1.56^{*}$ & $-1.48 \dagger$ & Geschlecht $(0=m, 1=w)$ & .19 & .07 & .20 \\
\hline BMI & $-.47 *$ & $-.47 *$ & $-.48^{*}$ & BMI & .21 & .21 & .20 \\
\hline Alter $\times$ Geschlecht & -.03 & -.03 & -.04 & Alter $\times$ Geschlecht & .03 & .04 & .03 \\
\hline Alter $\times$ BMI & .0005 & .0003 & -.0008 & Alter $\times$ BMI & .0005 & .0004 & .0002 \\
\hline Geschlecht $\times$ BMI & $.54 *$ & $.54 *$ & $.54 *$ & Geschlecht $\times$ BMI & -.36 & $-.37 \dagger$ & -.34 \\
\hline Alter $\times$ Geschlecht $\times$ BMI & .02 & .02 & .02 & Alter $\times$ Geschlecht $\times$ BMI & .005 & .005 & .005 \\
\hline Level $1 \times$ Level 2 & & & & Level $1 \times$ Level 2 & & & \\
\hline Bewegungsaktivität $\times$ Alter & -.006 & -.004 & -.006 & Bewegungsaktivität $\times$ Alter & .002 & -.006 & -.008 \\
\hline Bewegungsaktivität $\times$ Geschlecht & -.08 & .02 & -.07 & Bewegungsaktivität $\times$ Geschlecht & .12 & .28 & .10 \\
\hline Bewegungsaktivität $\times$ BMI & .06 & .07 & $.10 \dagger$ & Bewegungsaktivität $\times$ BMI & .006 & .005 & .01 \\
\hline
\end{tabular}

Anmerkungen: ${ }^{1)}=$ Bewegungsaktivität 1 Minute vor der Befindensabfrage, ${ }^{5}$ ) = Bewegungsaktivität 5 Minuten vor der Befindensabfrage, ${ }^{15)}=$ Bewegungsaktivität 15 Minuten vor der Befindensabfrage; $n=124, * * p<.01, * p<.05, \dagger p<.10$.

berechnet. Wie zu erwarten war, stiegen die Korrelationen mit Ausdehnung des analysierten Zeitfensters an. So korrelierte die Gesamtaktivität mit den gemittelten 1-Minutenwerten zu $r=.56(p<.01)$, mit den gemittelten 5-Minutenwerten $\mathrm{zu} r=.62(p<.01)$ und mit den gemittelten $15-$ Minutenwerten zu $r=.69(p<.01)$.

\section{Mehrebenenmodelle zur Vorhersage des Befindens im Alltag}

Anschließend wurden Mehrebenenmodelle zur Vorhersage der psychologischen Befindensvariablen EA/PA bzw. WA/NA berechnet. Die Ergebnisse für die drei verschiedenen Zeitfenster finden sich in Tabelle 1 (bei der Interpretation der Steigungskoeffizienten $(b)$ ist darauf zu achten, dass diese keine standardisierten Werte darstellen und daher in ihrer Größe nicht unmittelbar vergleichbar sind). Für die Variable EA/PA ergaben sich signifikante Effekte der Bewegungsaktivität (von $b=.44, p<.01$ für die 1 -minütige Bewegungsaktivität, bis $b=.58, p<.01$ für die 15-minütige Bewegungsaktivität), tendenziell signifikante Haupteffekte des Alters (für alle drei Zeitfenster $b=.08$, $p<.10$ ), teilweise signifikante Haupteffekte des Geschlechts $(b<-1.48, p<.10$ für die 1-Minuten und 15Minuten-Zeitfenster sowie $b=-1.56, p<.05$ für das 5-Minuten-Zeitfenster) sowie signifikante Haupteffekte des BMI (für alle drei Zeitfenster $b=-.47, p<.05$ ). Zudem zeigten sich in allen hier analysierten Zeitfenstern signifikante Interaktionen zwischen Geschlecht und BMI ( $b=$ $.54, p<.05)$, die darauf hindeuteten, dass der Zusammenhang zwischen BMI und EA/PA bei männlichen Versuchsteilnehmern signifikant negativ war $(b=-.47, p<.05)$, wohingegen er bei weiblichen Versuchsteilnehmern nicht signifikant war $(b=.052, p=.73)$.
Die Effekte lassen sich folgendermaßen interpretieren: Mit zunehmender Bewegungsaktivität steigen EA und PA an. Steigt die Bewegungsaktivität um 1000 Einheiten, führt dies zu einem Anstieg auf der Skala EA/PA um 0.44 Punkte bei 1-minütiger Bewegungsaktivität bzw. um 0.58 Punkte bei 15-minütiger Bewegungsaktivität. Die Größe dieses Effekts erscheint auf den ersten Blick als relativ gering, jedoch muss beachtet werden, dass langsames Gehen (ca. $3 \mathrm{~km} / \mathrm{h}$ ) von einer Minute Dauer zu einem Wert von etwa 5000 Einheiten führt. Entsprechend wäre eine Steigerung auf der Skala EA/PA um 2 bis 3 Punkte zu erreichen, wenn die Aktivität von Ruhe hin zu langsamem Gehen verlagert würde, bzw. um 3.5 bis 4.5 Punkte, wenn Treppen gestiegen würden.

Weiterhin besagt der Effekt des Alters, dass mit jedem weiteren Lebensjahr das Erleben von Energie und positivem Affekt um 0.08 Punkte zunimmt, und der Effekt des Geschlechts indiziert, dass sich die männlichen Teilnehmer um etwa 1.5 Punkte aktivierter beschreiben als die weiblichen Teilnehmer. Weiterhin scheint bei männlichen Probanden mit jedem weiteren BMI-Punkt das Erleben von EA/PA um 0.47 Punkte abzunehmen. Beispielsweise würde der Unterschied zwischen normalgewichtigen $(\mathrm{BMI} \approx$ 23 ) und adipösen Männern (BMI $\approx 30)$ auf der Skala EA/ PA etwa 3 Punkte betragen.

Die Analyse zur Vorhersage der Variable WA/NA erbrachte erwartungskonform kaum robuste Prädiktoren. Lediglich das Alter wies über alle hier analysierten Zeitfenster eine negative Assoziation mit WA/NA auf, d.h. mit jedem weiteren Lebensjahr wurde um 0.07 Punkte weniger WA/NA berichtet.

Eine genauere Inspektion der Daten ergab, dass die Level 1-Variable Bewegungsaktivität in allen Zeitfenstern 
die Grundannahme einer Normalverteilung nicht erfüllte, da überproportional viele Befindensabfragen mit NichtAktivität verbunden waren. Da eine Verletzung der Normalverteilungsannahme der Level 1-Prädiktoren in Mehrebenenmodellen zu unreliablen Signifikanzschätzungen führen kann (Raudenbush \& Bryk, 2002), wurden die Analysen mit dichotomisierten Bewegungsvariablen wiederholt. Die Einteilung in hohe vs. niedrige Aktivität erfolgte anhand von Mediansplits der Bewegungsvariablen.

Die Vorhersageleistung der Prädiktoren für die Variable EA/PA änderte sich dadurch nur geringfügig. ${ }^{4}$ Nach wie vor war die Assoziation zwischen Bewegungsaktivität und EA/PA signifikant $(b=.85, p<.05$ für das 1-MinutenIntervall, $b=1.46, p<.01$ für das 5-Minuten-Intervall und $b=1.68, p<.01$ für das 15-Minuten-Intervall). Ebenso blieben Alter, BMI und die Interaktion zwischen Geschlecht und BMI signifikante Prädiktoren. Darüber hinaus zeigte sich nun in den Analysen der 5- und 15-minütigen Bewegungsaktivität ein signifikanter Moderatoreffekt des BMI auf den Zusammenhang von Bewegung und EA/PA (für das 5-Minuten-Zeitfenster: $b=.23, p<$ .05 ; für das 15-Minuten-Zeitfenster: $b=.26, p<.05$ ). Wie weitere Analysen zeigten, bestand eine signifikante Korrelation zwischen dem Steigungskoeffizienten zur Vorhersage von EA/PA durch Bewegungsaktivität und dem BMI (für das 5-Minuten-Zeitfenster: $r=.19, p<.05$; für das 15-Minuten-Zeitfenster: $b=.20, p<.05)$, d. h. mit zunehmendem BMI wurde die Assoziation zwischen Bewegungsaktivität und EA/PA stärker.

Für die Variable WA/NA zeigten sich nach der modifizierten Analyse keine Veränderungen, d.h. es konnten bis auf das Alter-keine signifikanten Prädiktoren identifiziert werden.

\section{Diskussion}

Ziel der vorliegenden Studie war es, die Technik des ambulanten Monitoring auf eine häufig untersuchte gesundheitspsychologische Fragestellung anzuwenden und die methodische Herangehensweise zu illustrieren. Wir wollten zeigen, inwieweit ambulantes Monitoring dazu beitragen kann, Zusammenhänge zwischen Bewegungsaktivität und psychischem Befinden auch im Alltag nachzuweisen. In Übereinstimmung mit früheren Studien, die einen positiven Zusammenhang zwischen Bewegung und Parametern der positiven Aktiviertheit fanden (Ekkekakis, 2003; Ekkekakis et al., 2000; Thayer et al., 1993, 2003), konnten auch wir positive intraindividuelle Kovariationen zwischen alltäglichen Bewegungsepisoden und EA/PA beobachten. Dieser Zusammenhang war in jedem der hier analysierten Zeitfenster festzustellen. In Übereinstimmung mit Ekkekakis et al. (2000) oder Thayer et al. (2003) konnte

\footnotetext{
${ }^{4}$ Die Steigungskoeffizienten für die Bewegungsaktivität entsprechen nun der Veränderung auf den Skalen EA/PA, bzw. WA/ NA, wenn die Aktivität von gering ( - unterhalb des Medians) zu hoch (= oberhalb des Medians) geändert wurde.
}

für die Variable WA/NA kein Zusammenhang mit der Bewegungsaktivität nachgewiesen werden.

Die Ergebnisse zum Zusammenhang von Bewegung und EA/PA mögen zunächst trivial erscheinen. Ein höheres $\mathrm{Ma} ß$ an Bewegung ging intraindividuell mit gesteigerter subjektiv empfundener Aktiviertheit/positivem Affekt einher. Es sollte jedoch beachtet werden, dass die hier eingesetzten Items der Skala EA/PA auch Zustände wie leistungsfähig, müde oder dynamisch beschreiben, die allesamt nicht zwangsläufig mit Bewegungsaktivität assoziiert sein müssen. Es ist ebenso wahrscheinlich, dass andere Lebensstilfaktoren wie Schlafquantität und -qualität, Ernährung, Tagesform etc. das Beantwortungsverhalten auf diese Items beeinflusst haben könnten. Auch vor diesem Hintergrund lassen sich die teilweise nur moderaten Zusammenhänge von Bewegung und Befinden möglicherweise erklären.

Neben der signifikanten Assoziation zwischen Bewegung und EA/PA konnte diese Studie weitere interessante interindividuelle Effekte aufzeigen, die uns aus Laborstudien bisher nicht bekannt waren. Für die Variable EA/ PA zeigten sich Haupteffekte von Alter, BMI und Geschlecht, insofern, dass EA/PA mit zunehmendem Alter leicht anstieg, männliche Versuchsteilnehmer höhere Ausprägungen in EA/PA über den Tag hinweg berichteten als weibliche und mit zunehmendem BMI geringere Werte berichtet wurden. Der negative Zusammenhang mit dem BMI schien allerdings nur für die männlichen Probanden zu gelten, wie die signifikante Interaktion zwischen $\mathrm{Ge}$ schlecht und BMI erkennen ließ.

In den dichotomisierten Analysen zeigte sich darüber hinaus - zumindest für die längeren Zeitfenster - eine signifikante Interaktion zwischen Bewegungsaktivität und BMI, die darauf beruhte, dass mit zunehmendem BMI der Zusammenhang von Bewegung und EA/PA stärker wurde. Inhaltlich könnte dieser Zusammenhang dahingehend interpretiert werden, dass übergewichtige Personen besonders durch Alltagsbewegungen ihr generell geringer ausgeprägtes Empfinden von Aktiviertheit positiv beeinflussen könnten. Sicherlich sind hier weitere Replikationsstudien zu fordern, da dieser Moderatoreffekt unter Berücksichtigung kontinuierlicher Bewegungsdaten nicht gesichert werden konnte.

\section{Praktische Implikationen}

In neuerer Zeit ist postuliert worden, dass die mit Bewegung assoziierten affektiven Reaktionen für die Initiierung bzw. Aufrechterhaltung gesundheitsföderlicher körperlicher Aktivität wesentlich sein könnten (Abu-Omar \& Rütten, 2006; Ekkekakis et al., 2000). Unsere Studie liefert Hinweise dafür, dass auch körperliche Alltagsaktivität als aktivierend, leistungssteigernd, vitalisierend etc. wahrgenommen wird, was der Initiierung und Aufrechterhaltung anderer Formen körperlicher Aktivität dienlich sein könnte. Sicherlich ließe sich diese Hypothese nur durch prospektiv angelegte Studien adäquat überprüfen, in denen 
der Zusammenhang zwischen antezedenten alltäglichen Bewegungsepisoden und der zu einem späteren Zeitpunkt initiierten sportlichen Aktivität untersucht werden sollte. Besonders interessant scheint vor diesem Hintergrund auch der Befund unserer dichotomisierten Analysen zu sein, wonach mit zunehmendem BMI auch die psychischen Auswirkungen alltäglicher Bewegungsaktivitäten in positiver Weise zunehmen. Dieses Ergebnis widerspricht zunächst Befunden, wonach bei erhöhtem BMI die positive Stimmungslage mit zunehmender Bewegungsintensität abnimmt (Ekkekakis \& Lind, 2006), allerdings gilt es hier zu bedenken, dass in unserer Studie vorwiegend selbst gewählte Bewegungsaktivitäten von eher geringer Intensität anzutreffen waren. Es wäre nun interessant $\mathrm{zu}$ erfahren, inwieweit Personen, die besonders starke Assoziationen von Bewegung und EA/PA im Alltag zeigen, auch stärker von Interventionen zur Steigerung körperlicher Aktivität profitieren bzw. geringere DropoutRaten in Sportkursen zeigen.

\section{Probleme und mögliche Weiterentwicklung der Methodik}

Obgleich die Studie zeigen konnte, dass die Untersuchung des Zusammenhangs von Bewegungsaktivität und Befinden mit Hilfe der Technik des ambulanten Monitoring möglich und durchaus viel versprechend ist, sollen verschiedene Kritikpunkte nicht unerwähnt bleiben, die zu einer Weiterentwicklung der hier vorgestellten Methodik führen sollten.

Die hier gefundenen Assoziationen zwischen Bewegungsaktivität und spezifischen Befindensaspekten müssen in ihrer Größe als moderat eingestuft werden. Wie bereits erwähnt wurde, könnte eine Erklärung hierfür sicherlich in der starken Konfundierung von Tagesereignissen und weiteren Lebensstilfaktoren (Schlaf, Ernährung etc.) begründet sein. Es ist allerdings auch möglich, dass die hier eingesetzte Methodik auf Grund technischer Beschränkungen einen stärkeren Zusammenhang nicht zu entdecken erlaubte. So ist es möglich, dass die hier aggregierten Bewegungsepisoden bei vielen Probanden mit Phasen der Nichtbewegung stark konfundiert waren, bzw. die zeitliche Lokalisation der in diesen Zeitfenstern ausgeführten Bewegungen möglicherweise $\mathrm{zu}$ weit von der nachfolgenden Befindensabfrage entfernt war.

Eine mögliche Lösung dieses Problems könnte in zukünftigen Studien durch die Verwendung eines interaktiven ambulanten Monitorings erfolgen, bei dem die Befindlichkeitsabfrage an eine Onlineanalyse der Bewegungsdaten gekoppelt ist und beispielsweise nach einer gewissen Dauer und Intensität einer Bewegungseinheit das Befinden und die Art der Tätigkeit abgefragt wird (vgl. Myrtek, Foerster \& Brügner, 2001 für ein ähnliches Verfahren zur Rückmeldung von bewegungskorrigierten Herzfrequenzerhöhungen). Eine weniger aufwändige Alternative wäre es, zusätzlich zur Befindlichkeit Art und Dauer der Aktivität mittels PDA zu erfragen, um genauere
Informationen über die ausgeführte körperliche Aktivität zu erhalten. Eine solchermaßen verbesserte Methodik sollte in der Lage sein, wesentlich robustere Zusammenhänge von Bewegungsepisoden und Befindensvariablen hervorzubringen.

Ein weiteres messmethodisches Problem sollte angesprochen werden. Es kann hinterfragt werden, inwieweit die intraindividuellen Streuungen der Befindlichkeits- und Aktivitätswerte ausreichend groß waren, um substanzielle Kovariationen zwischen diesen Variablen wahrscheinlich werden zu lassen. Zudem war die Bewegungsaktivität in den hier analysierten Zeitfenstern nicht normalverteilt und viele Befindlichkeitsabfragen waren mit fehlender Bewegungsaktivität assoziiert, was das Auffinden substanzieller Kovariationen natürlich erschwert und die Güte der Auswertung mit Mehrebenenmodellen beeinträchtigt.

Das Problem eingeschränkter Varianzen könnte insbesondere eine Erklärung für den fehlenden Zusammenhang von Bewegung und wahrgenommener Anspannung/negativem Affekt darstellen. Ekkekakis (2003) weist zu Recht darauf hin, dass in Studien zur körperlichen Aktivität negativer Affekt generell seltener berichtet wird und entsprechend bewegungsassoziierte Veränderungen auf Grund von Bodeneffekten unwahrscheinlich werden. Dies mag auch in unserer Studie eine Rolle gespielt haben, da der Mittelwert der Skala WA/NA verglichen mit dem der Skala EA/PA doch sehr niedrig war. In diesem Zusammenhang sollten auch die Daten zur Typizitätseinschätzung des Tages kritisch beleuchtet werden. Etwa 1/3 der Probanden gab an, dass der Untersuchungstag eher bzw. ganz und gar untypisch verlief. Es ist plausibel anzunehmen, dass für die Versuchsteilnahme ein Tag gewählt wurde, der weniger emotionale oder beanspruchende Ereignisse erwarten lie $\beta$ und entsprechend zu Bodeneffekten in den Einschätzungen zum negativen Affekt geführt haben könnte.

Als mögliche Lösung des Problems eingeschränkter Varianzen wäre zu überlegen, die Untersuchung über längere Zeiträume (z. B. eine Woche) auszuweiten, um einen breiteren Variationsbereich in den subjektiven und behavioralen Variablen zu ermöglichen. Ebenfalls angedacht werden könnte die Implementierung einer Intervention zur Steigerung der Bewegungsaktivität oder die Untersuchung von Subgruppen, von denen angenommen werden kann, dass sie höhere Werte z. B. auf Skalen zum negativen Affekt aufweisen (z. B. Hochängstliche).

Weiterhin kann an dieser Studie der Ort der Ableitung der Bewegungsaktivität kritisiert werden. Wir wählten das Fußgelenk, um Aktivitäten der unteren Extremitäten (wie z.B. Treppensteigen, Gehen, Laufen) mit ausreichender Sensitivität zu erfassen. Zunehmend scheint sich in der Literatur jedoch die Ableitung an der Hüfte durchzusetzen, da diese Lokalisation metabolisch relevante Ganzkörperbewegungen spezifischer abbilden kann als Ableitungen an den Extremitäten (z. B. Trost et al., 2005). Entsprechend sollten zukünftige Untersuchungen das Aktivitätsmonitoring an der Hüfte vornehmen. 
Ein letzter kritischer Punkt betrifft die Frage der Kausalität der beobachteten Effekte. Obwohl die Bewegungsaktivität zeitlich der Befindensabfrage vorausging, dürfen die Befunde nicht notwendigerweise im Sinne einer Kausalitätsbeziehung interpretiert werden. Drittvariablen (z. B. das situative Setting oder personspezifische Merkmale) könnten den Zusammenhang zwischen Bewegungsaktivität und EA/PA moderiert haben. So wäre es beispielsweise denkbar, dass unmittelbar nach einer Nahrungsaufnahme sowohl die energetische Aktiviertheit abnimmt, als auch die körperliche Aktivität reduziert wird, ohne dass ein kausaler Zusammenhang zwischen den Variablen bestehen muss. Gleichfalls wäre es denkbar, dass Personen, die sportlich aktiv sind, sich auch im Alltag mehr bewegen und sich gleichzeitig aktiver, dynamischer etc. fühlen.

Um die Wirkungsrichtung in weiteren Studien einzugrenzen, scheint es unerlässlich, Bedingungsmanipulationen einzuführen, wobei beispielsweise die Auswirkung einer Intervention zur Steigerung alltäglicher körperlicher Aktivität auf das Befinden getestet werden könnte. Darüber hinaus wäre es interessant, weitere Moderatoren des Bewegungs-Befindens-Zusammenhangs zu identifizieren. Mögliche Kandidaten sind z. B. sportliche Aktivität, Sedentarismus, Ernährung oder Persönlichkeitseigenschaften wie Neurotizismus oder Extraversion.

\section{Schlussfolgerung}

Abschließend bleibt zu vermerken, dass die vorliegende Studie trotz der angesprochenen Probleme und methodischen Kritikpunkte zeigt, dass die alltagsnahe Erhebung des Zusammenhangs von körperlicher Bewegung und Befinden viel versprechend ist. Die Ergebnisse sind weitgehend kompatibel zu denen unter kontrollierten Bedingungen durchgeführter Studien und zeigen, dass auch im Alltag durchgeführte Bewegungsaktivität von kurzer Dauer mit spezifischen Aspekten des Befindens assoziiert ist. Darüber hinaus konnten wir zeigen, dass demographische Variablen, wie Alter, Geschlecht oder BMI, den Grad der wahrgenommenen positiven Aktiviertheit beeinflussen bzw. den Zusammenhang zwischen Bewegungsaktivität und EA/AP moderieren könnten. Wir sind zuversichtlich, dass eine Weiterentwicklung dieses Ansatzes im Sinne der oben beschriebenen Entwicklungslinien zu neuen Erkenntnissen in diesem Bereich beitragen kann.

\section{Literatur}

Abu-Omar, K. \& Rütten, A. (2006). Sport oder körperliche Aktivität im Alltag? Zur Evidenzbasierung von Bewegung in der Gesundheitsförderung. Bundesgesundheitsblatt - Gesundheitsforschung - Gesundheitsschutz, 49, 1162-1168.

Arent, S. M., Landers, D. M. \& Etnier, J. L. (2000). The effects of exercise on mood in older adults: A meta-analytic review. Journal of Aging and Physical Activity, 8, 407-430.

Craft, L. L. \& Landers, D. M. (1998). The effects of exercise on clinical depression and depression resulting from mental illness: A meta-analysis. Journal of Sport \& Exercise Psychology, 20, 339-357.
Ebner-Priemer, U., Linehan, M. M. \& Bohus, M. (2006). Ambulantes psychophysiologisches Monitoring in der psychiatrischen Forschung - Eine Untersuchung der BorderlinePersönlichkeitsstörung. In U. Ebner-Priemer (Hrsg.), Ambulantes psychophysiologisches Monitoring - neue Perspektiven und Anwendungen (S. 113-134). Frankfurt: Lang.

Ekkekakis, P. (2003). Pleasure and displeasure from the body: Perspectives from exercise. Cognition and Emotion, 17, 213 239.

Ekkekakis, P., Hall, E. E., van Landuyt, L. M. \& Petruzello, S. J. (2000). Walking in (affective) circles: Can short walks enhance affect? Journal of Behavioural Medicine, 23, 245 275.

Ekkekakis, P. \& Lind, E. (2006). Exercise does not feel the same when you are overweight: The impact of self-selected and imposed intensity on affect and exertion. International.Journal of Obesity, 30, 652-660.

Fahrenberg, J., Leonhart, R. \& Foerster, F. (2002). Alltagsnahe Psychologie. Bern: Huber.

Fahrenberg, J., Myrtek, M., Pawlik, K. \& Perrez, M. (2007). Ambulantes Assessment - Verhalten im Alltagskontext erfassen. Psychologische Rundschau, 58, 12-23.

Focht, B. C., Gauvin, L. \& Rejeski, W. J. (2004). The contribution of daily experiences and acute exercise to fluctuations in daily feeling states among older, obese adults with knee osteoarthritis. Journal of Behavioral Medicine, 27, 101-121.

Foerster, F. \& Fahrenberg, J. (2000). Motion pattern and posture: Correctly assessed by calibrated accelerometers. Behavior Research Methods, Instruments \& Computers, 32, 450457.

Fuchs, R. (2003). Sport, Gesundheit und Public Health. Göttingen: Hogrefe.

Guinhouya, C. B., Hubert, H., Dupont, G. \& Durocher, A. (2005). Relationship between the MTI accelerometer (Actigraph) counts and running speed during continuous and intermittent exercise. Journal of Sports Science and Medicine, $4,534-542$.

Hall, E. H., Ekkekakis, P. \& Petruzello, S. J. (2002). The affective beneficence of vigorous exercise revisited. British Journal of Health Psychology, 7, 47-66.

Imhof, M. (1998). Erprobung der deutschen Version der Adjektiv-Checkliste nach Thayer (1989) zur Erfassung der aktuellen Aktiviertheit. Zeitschrift für Differentielle und Diagnostische Psychologie, 19, 179-168.

Käppler, C., Brügner, G. \& Fahrenberg, J. (2001). Pocketcomputer-unterstûtztes Assessment mit MONITOR: Befindlichkeit im Alltag, Methodenakzeptanz und die Replikation des Retrospektionseffektes. Zeitschrift fûr Differentielle und Diagnostische Psychologie, 22, 249-266.

Keller, F. (2003). Analyse von Längsschnittdaten: Auswertungsmöglichkeiten mit hierarchischen linearen Modellen. Zeitschrift für Klinische Psychologie und Psychotherapie, 32, 51-61.

Krohne, H. W., Egloff, B., Kohlmann, C. \& Tausch, A. (1996). Untersuchungen mit einer deutschsprachigen Version der „Positive and Negative Affect Schedule“ (PANAS). Diagnostica, 42, 139-156.

Matthews, C. E., Ainsworth, B. E., Thompson, R. W. \& Basset, D. R., Jr. (2002). Sources of variance in daily physical activity level as measured by an accelerometer. Medicine and Science in Sports and Exercise, 34, 1376-1381.

Myrtek, M., Foerster, F. \& Brügner, G. (2001). Freiburger Monitoring System (FMS). Ein Daten-Aufnahme- und Auswertungssystem für Untersuchungen im Alltag: Emotionale Beanspruchung, Körperlage, Bewegung, EKG, subjektives Befinden, Verhalten. Frankfurt/M.: Lang.

National Institutes of Health (1996). NIH consensus development panel on physical activity and cardiovascular health: Physical activity and cardiovascular health. Journal of the American Medical Association, 276, 241-246. 
Nezlek, J. B., Schröder-Abé, M. \& Schütz, A. (2006). Mehrebenenanalysen in der psychologischen Forschung. Vorteile und Möglichkeiten der Mehrebenenmodellierung mit Zufallskoeffizienten. Psychologische Rundschau, 57, 213-223.

Otto, J. \& Stemmann, O. (1991). Befindlichkeitsveränderungen durch abgestufte, moderate körperliche Aktivierung. Zeitschrift für experimentelle und angewandte Psychologie, 38, 264-278.

Pate, R. R., Pratt, M., Blair, S. N., Haskell, W. L., Macera, C. A., Bouchard, C., Buchner, D., Ettinger, W., Heath, G.W., King, A. C., Kriska, A., Leon, A. S., Marcus, B. H., Morris, J., Paffenbarger, R. S. Jr, Patrick, K., Pollock, M. L., Rippe, J. M., Sallis, J. \& Wilmore, J. H. (1995). Physical activity and public health: A recommendation from the Centers for $\mathrm{Di}$ sease Control and Prevention and the American College of Sports Medicine. Journal of the American Medical Association, 273, 402-407.

Pinheiro, J., Bates, D., DebRoy, S. \& Sarkar, D. the R Core team. (2007). nlme: Linear and Nonlinear Mixed Effects Models. $R$ package version 3.1-83.

Puetz, T. W., O’Connor, P. J. \& Dishman, R. K. (2006). Effects of chronic exercise on feelings of energy and fatigue: A quantitative synthesis. Psychological Bulletin, 132, 866-876.

R Development Core Team (2006). R: A Language and Environment for Statistical Computing. R Foundation for Statistical Computing, Vienna, Austria.

Raudenbush, S. W. \& Bryk, A. S. (2002). Hierarchical Linear Models. Applications and Data Analysis Methods (2nd ed.). London: Sage.

Reed, J. \& Ones, J. (2006). The effect of acute aerobic exercise on positive activated affect: A meta-analysis. Psychology of Sport and Exercise, 7, 477-514.

Schlicht, W. (1993). Psychische Gesundheit durch Sport - Realität oder Wunsch: Eine Meta-Analyse. Zeitschrift für Gesundheitspsychologie, 1, 65-81.

Schwartz, J. E. \& Stone, A. A. (1998). Strategies for analyzing ecological momentary data. Health Psychology, 17, 6-16.
Thayer, R. E. (1978). Toward a Psychological Theory of Multidimensional Activation (Arousal). Motivation and Emotion, $2,1-34$.

Thayer, R. E. (1989). The biopsychology of mood and arousal. New York: Oxford University Press.

Thayer, R. E., Godes, O., Lobato, N. E., Serrano, M., Hernandez, J. \& Culver, S. (2003). Walking, energy, and mood are interrelated: An important health indication. Poster presented at the American Psychological Association, Toronto.

Thayer, R. E., Peters, D. P., Takahashi, P. J. \& Birkhead-Flight, A. M. (1993). Mood and behaviour (smoking and sugar snacking) following moderate exercise: A partial test of selfregulation theory. Personality and Individual Differences, 14, 97-104.

Trost, S. G., McIver, K. L. \& Pate, R. R. (2005). Conducting accelerometer-based activity assessment in field-based research. Medicine and Science in Sports and Exercise, 37, 531-543.

Trost, S. G., Pate, R. R., Freedson, P. S., Sallis, J. F. \& Taylor, W. C. (2000). Using objective physical activity measures with youth: How many days of monitoring are needed? Medicine and Science in Sports and Exercise, 32, 426-431.

Watson, D., Clark, L. A. \& Telegen, A. (1988). Development and validation of brief measures of positive and negative affect: the PANAS scales. Journal of Personality and Social Psychology, 54, 1063-1070.

Yik, M. S., Russell, J. A. \& Feldman Barrett, L. (1999). Structure of self-reported current affect: Integration and beyond. Journal of Personality and Social Psychology, 77, 600-619.

Prof. Dr. Andreas Schwerdtfeger

Staudinger Weg 9

55099 Mainz

E-Mail: aschwerd@uni-mainz.de 